\title{
Histone deacetylase adaptation in single ventricle heart disease and a young animal model of right ventricular hypertrophy
}

\author{
Weston W. Blakeslee', Kimberly M. Demos-Davies², Douglas D. Lemon², Katharina M. Lutter², Maria A. Cavasin²,
} Sam Payne ${ }^{2}$, Karin Nunley ${ }^{2}$, Carlin S. Long ${ }^{3}$, Timothy A. McKinsey ${ }^{2}$ and Shelley D. Miyamoto ${ }^{4}$

BACKGROUND: Histone deacetylase (HDAC) inhibitors are promising therapeutics for various forms of cardiac diseases. The purpose of this study was to assess cardiac HDAC catalytic activity and expression in children with single ventricle (SV) heart disease of right ventricular morphology, as well as in a rodent model of right ventricular hypertrophy (RVH).

METHODS: Homogenates of right ventricle (RV) explants from non-failing controls and children born with a SV were assayed for HDAC catalytic activity and HDAC isoform expression. Postnatal 1-day-old rat pups were placed in hypoxic conditions, and echocardiographic analysis, gene expression, HDAC catalytic activity, and isoform expression studies of the RV were performed.

RESULTS: Class I, $\| \mathrm{a}$, and Ilb HDAC catalytic activity and protein expression were elevated in the hearts of children born with a SV. Hypoxic neonatal rats demonstrated RVH, abnormal gene expression, elevated class I and class IIb HDAC catalytic activity, and protein expression in the RV compared with those in the control.

CONCLUSIONS: These data suggest that myocardial HDAC adaptations occur in the SV heart and could represent a novel therapeutic target. Although further characterization of the hypoxic neonatal rat is needed, this animal model may be suitable for preclinical investigations of pediatric RV disease and could serve as a useful model for future mechanistic studies.

$\mathbf{R}$ ight ventricular hypertrophy (RVH) and pulmonary hypertension $(\mathrm{PH})$ are common sequelae in pediatric patients with various forms of congenital heart diseases. On the severe end of the spectrum are children with single ventricle (SV) heart disease, especially those with a single right ventricle (RV), such as hypoplastic left heart syndrome. $\mathrm{SV}$ is a rare but important form of hypoxic congenital heart disease that is fatal without intervention (1). Current treatment strategies for infants born with a SV include a threestage surgical palliation or primary heart transplantation.
Primary heart transplant is significantly limited by donor availability. Although there have been advancements in perioperative and surgical approaches for SV, in the largest prospective study to date, $32 \%$ of those managed with surgical palliation died or were transplanted before 1 year of age (2). Whereas there are many causes of poor outcome in patients born with a SV, systemic right ventricular dysfunction, for which there are no proven therapies, is a risk factor for death and listing for transplant (3-6).

Histone deacetylases (HDACs) are epigenetic enzymes that function canonically through the removal of acetyl groups from lysine residues within nucleosomal histone tails. The 18 mammalian HDACs are categorized into 4 classes: class I (HDACs 1, 2, 3, and 8), class II (class IIa: HDACs 4, 5, 7, and 9; class IIb: HDACs 6 and 10), class III (SIRT1-7), and class IV (HDAC11). Class III HDACs are also known as sirtuins and use $\mathrm{NAD}^{+}$as a cofactor, whereas HDACs 1-11 employ $\mathrm{Zn}^{2+}$ as a cofactor for catalytic activity. Class IIa HDACs are thought to be protective in the setting of heart failure (HF) because of their ability to bind to and inhibit the transcriptional activity of myocyte enhancer factor 2 (ref. 7). Elevated catalytic activity of sirtuins appears to be beneficial in failing hearts (8), whereas elevated catalytic activity of class I and IIb HDACs is thought to be maladaptive $(9,10)$.

Two HDAC inhibitors, Vorinostat (suberoylanilide hydroxamic acid, Zolinza) and Romidepsin (Istodax), are Food and Drug Administration-approved to treat cutaneous T-cell lymphoma. These and other HDAC inhibitors are currently in clinical trials, investigating efficacy in a variety of oncologic and non-oncologic diseases. HDAC inhibition has beneficial effects in preclinical models of adult HF, reducing cardiac hypertrophy (11) and fibrosis (12), and suppressing the fetal gene program associated with adverse cardiac remodeling (13). These findings suggest that HDACs could be therapeutically targeted for the treatment of adult human HF (14). However, nothing is known about the roles of HDACs in pediatric HF.

The purpose of this study was to examine the catalytic activity and protein expression of HDACs in pediatric patients born with a SV with a single RV, and to characterize

\footnotetext{
${ }^{1}$ Department of Pharmacology, University of Colorado Denver Anschutz Medical Campus, Aurora, Colorado; ${ }^{2}$ Division of Cardiology, Department of Medicine, University of Colorado Denver Anschutz Medical Campus, Aurora, Colorado; ${ }^{3}$ Division of Cardiology, Department of Medicine, Denver Health and Hospital Authority, Denver, Colorado; ${ }^{4}$ Department of Pediatrics, University of Colorado School of Medicine, Children's Hospital Colorado, Aurora, Colorado. Correspondence: Shelley D. Miyamoto (shelley. miyamoto@childrenscolorado.org) 
a neonatal animal model with the potential to elucidate molecular mechanisms controlling pathological remodeling of the pediatric RV. We hypothesized that pediatric patients born with a SV would exhibit elevated HDAC catalytic activity, and that hypoxia would result in elevated HDAC catalytic activity coincident with adverse remodeling in RVs of neonatal rat.

\section{METHODS}

This study used heart tissue samples from pediatric patients $(<18$ years of age) who donated their hearts to the University of Colorado Institutional Review Board-approved pediatric heart tissue bank (informed consent is obtained from all patients). Patients included males and females of all races and ethnic background undergoing heart transplantation at the Children's Hospital Colorado. Nonfailing (NF) control hearts were obtained from pediatric donors $(<18$ years of age) with structurally normal hearts and those with normal heart function whose hearts could not be placed for technical reasons, usually size or blood-type mismatch. SV tissue was obtained from explanted hearts of patients with SV physiology and a morphologic single RV. Patients with a single left ventricle (LV) or those with indeterminate morphology of the SV were excluded. All heart tissues were rapidly flash-frozen in the operating room immediately after removal from the subject. A detailed description of patients included in this study is outlined in Table 1.

\section{Histone Deacetylase Catalytic Activity Assays}

Measurement of HDAC catalytic activity was performed as previously described (15). Reagents were purchased from indicated vendors. HDAC inhibitor, Trichostatin A (Sigma, Rowville, VIC, Australia), was diluted in dimethylsulfoxide. Synthetic HDAC substrates are as follows: class I HDAC substrate (custom synthesis by Genscript, Piscataway, NJ, USA), class IIa HDAC substrate (I-1985; Bachem), and class I/IIb HDAC substrate (I-1875; Bachem, Torrance, CA, USA). Trypsin, Triton X-100, and dimethylsulfoxide were obtained from Sigma. 7-Amino-4-methylcoumarin was obtained from Alfa Aesar (Tewksbury, MA, USA).

Frozen explants from human pediatric RV were prepared in phosphate-buffered saline ( $\mathrm{pH} 7.4)$ containing $0.5 \%$ Triton X-100, $300 \mathrm{mM} \mathrm{NaCl}$, and HALT protease/phosphatase inhibitor cocktail (ThermoFisher, Waltham, MA, USA) using a Bullet Blender homogenizer (Next Advance, Averill Park, NY, USA). Protein concentrations were determined with the BCA Protein Assay Kit (ThermoFisher). Incubation of tissue lysates with class-selective HDAC substrates results in a cleavable 7-Amino-4-methylcoumarin product. 7-Amino-4-methylcoumarin fluorescence was measured using a BioTeK Synergy 2 plate reader, with excitation and emission filters of 360 and $460 \mathrm{~nm}$, respectively. Background fluorescence from buffer blanks was subtracted from raw signals, and data were normalized as needed using appropriate controls.

\section{Immunoblotting}

Immunoblotting was performed as previously described (16). Briefly, pediatric human and neonatal rat RV homogenates were prepared and concentrations quantified as above for HDAC catalytic activity assay. Proteins were resolved with sodium dodecyl sulfatepolyacrylamide gel electrophoresis, transferred to nitrocellulose membranes (Bio-Rad, Hercules, CA), and probed with antibodies for HDAC1 (Cell Signaling Technology, Danvers, MA, USA, 5356), HDAC2 (Cell Signaling Technology, 5113), HDAC3 (Cell Signaling Technology, 3949), HDAC4 (Cell Signaling Technology, 5392), HDAC5 (Cell Signaling Technology, 2082), HDAC6 (Santa Cruz Biotechnology, Dallas, TX, USA, 11420), HDAC7 (Cell Signaling Technology, 2882), and calnexin (Santa Cruz Biotechnology, 11397).

\section{Animal Model}

The pediatric neonatal rat RV hypertrophy model was adapted from a previous study (17). Timed-pregnant E17 Sprague-Dawley rats were obtained from Charles River Laboratories (Waltham, MA, USA). Rat mothers birthed their litters at Denver altitude and equilibrated for 1 day. Postnatal day-1 normoxic animals were placed in a hypobaric chamber simulating a sea-level altitude ( $21 \%$ oxygen), whereas postnatal day-1 hypoxic animals were placed in a hypobaric

Table 1. Patient demographics

\begin{tabular}{|c|c|c|c|c|c|c|c|c|c|c|}
\hline Study ID & $\begin{array}{l}\text { Age } \\
\text { (years) }\end{array}$ & Sex & $\begin{array}{l}\text { HDAC } \\
\text { activity }\end{array}$ & $\begin{array}{c}\text { HDAC } \\
\text { Westerns }\end{array}$ & Inotrope & Digoxin & ACEI & $\mathrm{BB}$ & Diuretic & Palliation history/ indication for transplant \\
\hline NF1 & 13 & M & $x$ & $x$ & Y & $\mathrm{N}$ & $\mathrm{N}$ & $\mathrm{Y}$ & $\mathrm{N}$ & N \\
\hline NF2 & 8 & $\mathrm{~F}$ & $x$ & $x$ & $\mathrm{~N}$ & $\mathrm{~N}$ & $\mathrm{~N}$ & $\mathrm{~N}$ & $\mathrm{~N}$ & $\mathrm{~N}$ \\
\hline NF3 & 7 & M & $x$ & $x$ & $\mathrm{~N}$ & $\mathrm{~N}$ & $\mathrm{~N}$ & $\mathrm{~N}$ & $\mathrm{~N}$ & $\mathrm{~N}$ \\
\hline NF4 & 1.4 & M & $x$ & $x$ & $\mathrm{~N}$ & $\mathrm{~N}$ & $\mathrm{~N}$ & $\mathrm{~N}$ & $\mathrm{~N}$ & $\mathrm{~N}$ \\
\hline NF5 & 12 & $M$ & $x$ & $x$ & $\mathrm{~N}$ & $\mathrm{~N}$ & $\mathrm{~N}$ & $\mathrm{~N}$ & $\mathrm{~N}$ & $\mathrm{~N}$ \\
\hline SV1 & 0.1 & $\mathrm{~F}$ & $x$ & $x$ & $\mathrm{~N}$ & $\mathrm{~N}$ & $\mathrm{~N}$ & $\mathrm{~N}$ & $Y$ & $\begin{array}{l}\text { No palliation, on PGE/primary transplant, RV } \\
\text { dilation }\end{array}$ \\
\hline SV2 & 0.1 & $M$ & $x$ & $x$ & $\mathrm{~N}$ & $\mathrm{~N}$ & $\mathrm{~N}$ & $\mathrm{~N}$ & $Y$ & $\begin{array}{l}\text { Aortic valvuloplasty and atrial septal stent on } \\
\text { PGE/not a candidate for additional surgical } \\
\text { palliation, ventilator-dependent }\end{array}$ \\
\hline SV6 & 0.1 & $M$ & $x$ & $x$ & $\mathrm{~N}$ & $Y$ & $Y$ & $\mathrm{~N}$ & $Y$ & $\begin{array}{l}\text { No palliation, on PGE/primary transplant, RVH } \\
\text { and RV dilation }\end{array}$ \\
\hline
\end{tabular}

ACEl, angiotensin-converting enzyme inhibitor; BB, beta-blocker (the non-selective beta-blocker labetalol was used in NF1); F, female; HDAC, histone deacetylase; M, male; NA, not available; NF, non-failing control; PDA, patent ductus arteriosus; PDE, phosphodiesterase; PGE, prostaglandin E1, used to maintain ductus arteriosus patency; RV, right ventricle; RVH, right ventricular hypertrophy; SV, single ventricle

Inotropes included dopamine and norepinephrine. 


\section{Articles | Blakeslee et al.}

chamber simulating 18,000 feet ( $10 \%$ oxygen) for 7 days. Neonatal rats were anesthetized with isoflurane and killed by thoracotomy. Hearts were flushed with saline through the aorta, atria were removed, and RVs were carefully separated from the LV and septum before flash-freezing in liquid nitrogen.

\section{Cardiac Imaging}

Echocardiographic analysis was performed on the neonatal rats using a Vevo2100 system equipped with a MS400 18-38 mHz transducer (VisualSonics, Toronto, ON, Canada), as previously described (9).

\section{Real-Time Polymerase Chain Reaction}

Real-time polymerase chain reactions (RT-PCR) were performed as previously described (18). Total RNA was extracted using a mirVana kit (Ambion, Austin, TX), RNA was reverse-transcribed into complementary DNA using I-script (Bio-Rad), and Power SYBR Green PCR Master Mix was (Applied Biosystems/Life Technologies, Carlsbad, CA) used in the RT-PCR reactions. Reactions were performed using the ABI 7300 System (ThermoFisher, Waltham, MA, USA). Primer sequences are indicated below:

ANF: Forward 5'-GCGAAGGTCAAGCTGCTT-3'; Reverse 5'-C TGGGCTCCAATCCTGTCAAT-3'.

B-type natriuretic peptide: Forward $5^{\prime}$-GGTGCTGCCCCAG ATGATT-3'; Reverse 5'-GGTGCTGCCCCAGATGATT-3'.

Sarcoplasmic reticulum calcium-ATPase 2a: Forward $5^{\prime}$-GGC CAGATCGCGCTACA; Reverse 5'-GGGCCAATTAGAGAGCAG GTTT-3'.

\section{Data Analysis}

GraphPad Prism software (LaJolla, CA, USA) was used to generate graphs and analyze data. Student's $t$-test with Welch's correction or ANOVA with Bonferroni's post-test $(P<0.05)$ was used to determine statistical differences between groups.

\section{RESULTS}

\section{Patient Characteristics}

The age range of the NF controls was $1.4-14$ years, with $17 \%$ female. The SV group was composed of infants less than 3 months of age, with $17 \%$ female. The indication for transplantation for the SV group is outlined in Table 1, and it encompasses those undergoing primary transplant (listed for transplant shortly after birth before surgical palliation) and includes one patient who failed an attempt at interventional palliation.

\section{Cardiac HDAC Catalytic Activity is Increased in Pediatric Patients Born with a SV}

Eleven zinc-dependent HDACs are grouped into four classes (Figure 1a). HDAC catalytic activity is yet to be quantified in the human heart. To investigate whether pediatric patients born with a SV have altered HDAC catalytic activity, RV homogenates from pediatric NF controls and pediatric patients born with a SV were incubated with HDAC classspecific fluorescent substrates. A significant increase in class I, IIa, and IIb HDAC catalytic activity was observed in RVs of patients born with a SV relative to RVs of NF control (Figure 1b). Class IIb HDAC catalytic activity was most markedly increased ( 3.5 -fold), followed by a twofold increase in class I HDAC catalytic activity, and a modest, but statistically significant, increase in class IIa HDAC catalytic activity. These observations are consistent with reported increases in a

\begin{tabular}{|c|c|c|c|}
\hline Class I & Class Ila & Class IIb & Class IV \\
\hline HDAC1 & HDAC4 & HDAC6 & HDAC11 \\
\hline HDAC2 & HDAC5 & HDAC10 & \\
\hline HDAC3 & HDAC7 & & \\
\hline HDAC8 & HDAC9 & & \\
\hline
\end{tabular}

b

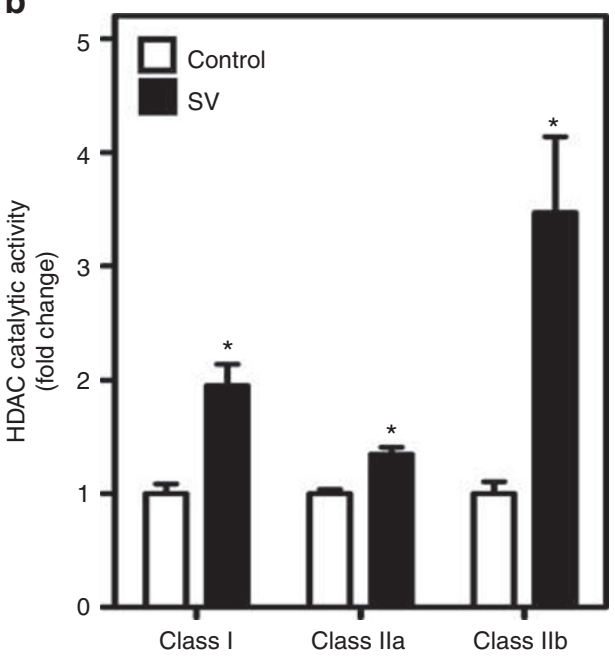

Figure 1. HDAC catalytic activity is elevated in the RV of children with a SV. (a) Classifications of zinc-dependent HDACs. (b) HDAC catalytic activity: RV tissue from non-failing pediatric and SV hearts was homogenized in a mild lysis buffer and incubated with HDAC class $\mathrm{I}-$, Ila-, and Ilb-specific substrates. All classes of HDACs displayed elevated catalytic activity relative to control. Results are displayed as the mean with $\mathrm{SE} ; N=6$ per group, ${ }^{*} P<0.05$ vs. controls. HDAC, histone deacetylase; RV, right ventricle; SV, single ventricle.

HDAC catalytic activity in hearts of adult rats with RVH due to $\mathrm{PH}(15)$.

Protein Expression of Distinct HDAC Isoforms is Elevated in the Hearts of Pediatric patients born with a SV

As all classes of HDACs measured in the enzymatic assay exhibited elevated catalytic activity in RVs of patients born with a SV, we investigated whether protein expression of HDACs belonging to class I, IIa, and IIb was concomitantly augmented. Protein expression of HDAC1-7 was measured by immunoblotting (Figure 2a), and quantified by normalization to a house-keeping protein, calnexin (Figure 2b). Expression of class I HDAC-1, -2, and -3 was elevated, and it reached a statistical significance in RVs of patients born with a SV compared with NF controls. Of the class IIa HDACs, only HDAC5 expression was significantly elevated in RVs of patients born with a SV. Expression of class IIb HDAC6 was not increased in RVs of patients born with a SV.

Activation of Cardiac HDACs in a Hypoxic Neonatal Rat Model $\mathrm{RVH}$ and failure is a common phenotype in pediatric patients with $\mathrm{PH}$ and various congenital heart lesions, including SV. In 


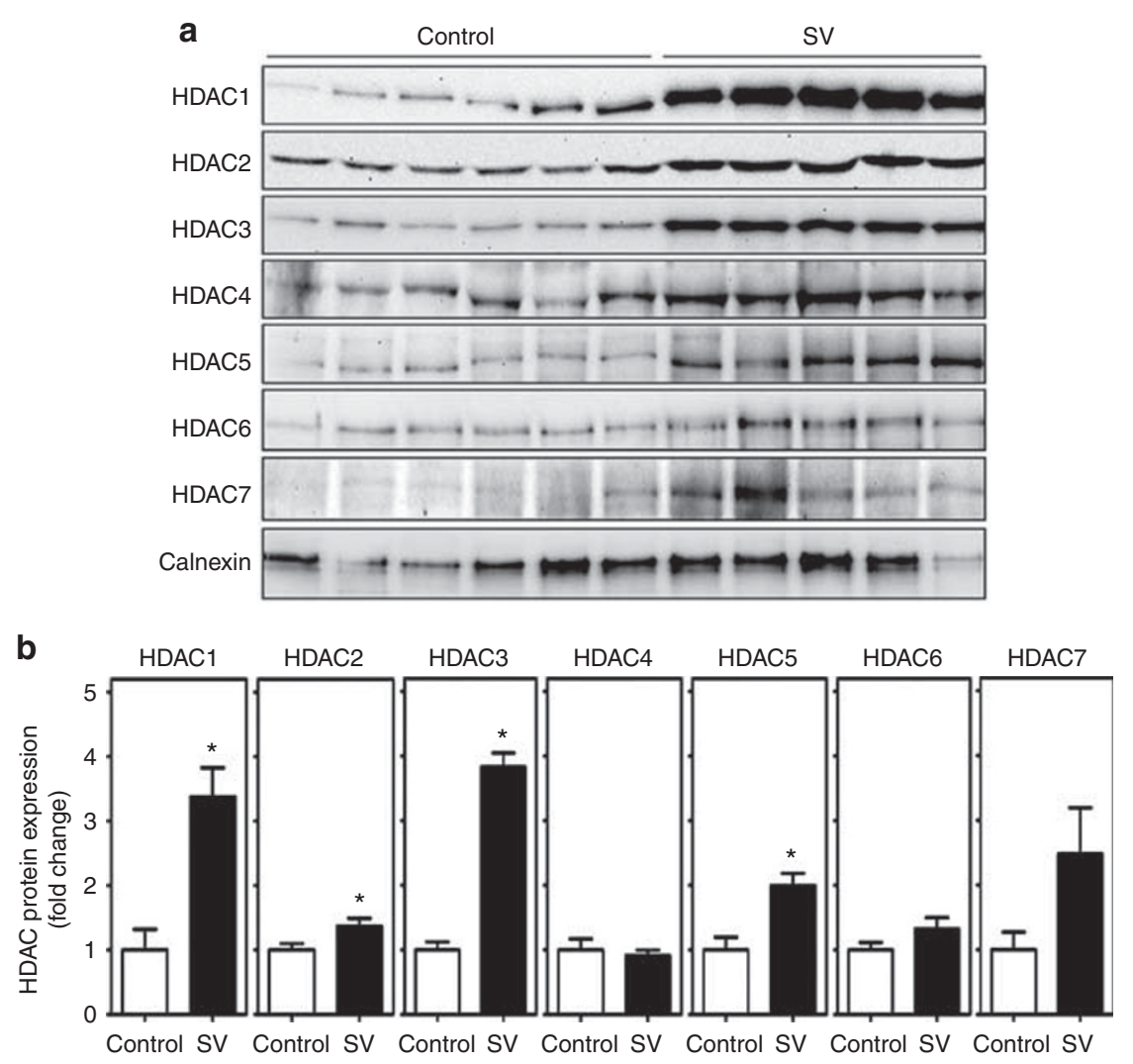

Figure 2. HDAC protein expression is elevated in pediatric RVs from patients born with a SV. (a) Immunoblot analysis was performed with the same homogenates used for the HDAC catalytic activity assays employing antibodies specific for class I HDACs (HDAC1-3), class Ila HDACs (HDAC4, 5, and 7), and class Ilb HDAC6. (b) Densitometry was used to quantify immunoblot signals; ${ }^{*} P<0.05$ vs. controls. HDAC, histone deacetylase; RV, right ventricle; SV, single ventricle.

an effort to recreate elements of pediatric $\mathrm{RVH}$, neonatal rats were placed in a hypobaric chamber simulating 18,000 feet in elevation and $10 \% \mathrm{O}_{2}$ for 7 days. Control animals were simultaneously housed at a sea-level altitude $\left(21 \% \mathrm{O}_{2}\right)$ for 7 days (Figure 3a). The use of neonatal rats, as opposed to mice, facilitated echocardiographic assessment of RV hypertrophy and pulmonary artery blood flow.

In response to 7 days of hypobaric hypoxia, thickness of the free wall of the neonatal rat RV was significantly increased in both systole and diastole compared with sea-level controls, as quantified by M-mode echocardiography (Figure $3 \mathbf{b}, \mathbf{c}$ ). Assessment of heart chamber remodeling by B-mode parasternal short-axis evaluation demonstrated septal wall flattening and chamber dilation in the RVs of hypoxic neonatal rats (Figure $3 \mathbf{b}$, lower images). Septal wall flattening occurred in $100 \%$ of the hypoxic animals.

Pulmonary artery blood flow was assessed using color Doppler and pulse-wave Doppler. Hypoxic neonatal rats exhibited a significant decrease in pulmonary artery acceleration time compared with sea-level controls. Pulmonary valve velocity-time integral and pulmonary valve peak velocity were also significantly decreased in hypoxic rats (Figure $\mathbf{3 d - f}$ and data not shown); pulmonary artery acceleration time, pulmonary valve peak velocity, and PV velocity-time integral are well-accepted measurements of PH in humans $(19,20)$. These findings suggest that $\mathrm{PH}$ exists in neonatal rats exposed to 7 days of hypobaric hypoxia.

Morphometric analysis performed at the time of necropsy confirmed significant RV hypertrophy in neonatal rats exposed to hypobaric hypoxia. After 7 days of hypobaric hypoxia, the ratio of RV-to-LV+septum (S) was 1.5-fold higher in hypoxic rats compared with sea-level control animals (Figure 4a).

Myocardial failure in humans is characterized by activation of a "fetal" gene program, as embryonically expressed genes are reactivated (7). This gene program is indicative of ventricular remodeling and is typified by increases in expression of B-type natriuretic peptide and atrial natriuretic peptide, and a coordinate decrease in sarcoplasmic reticulum calcium-ATPase 2a expression. Quantitative PCR revealed that atrial natriuretic peptide and B-type natriuretic peptide mRNA levels were elevated three- and fivefold, respectively, whereas sarcoplasmic reticulum calcium-ATPase $2 \mathrm{a}$ expression was significantly attenuated in the RVs of hypoxic neonatal rat compared with RVs of sea-level control (Figure 4b-d).

HDAC catalytic activity was subsequently quantified in homogenates of RVs from the sea level and hypoxic neonatal 


\section{Articles | Blakeslee et al.}
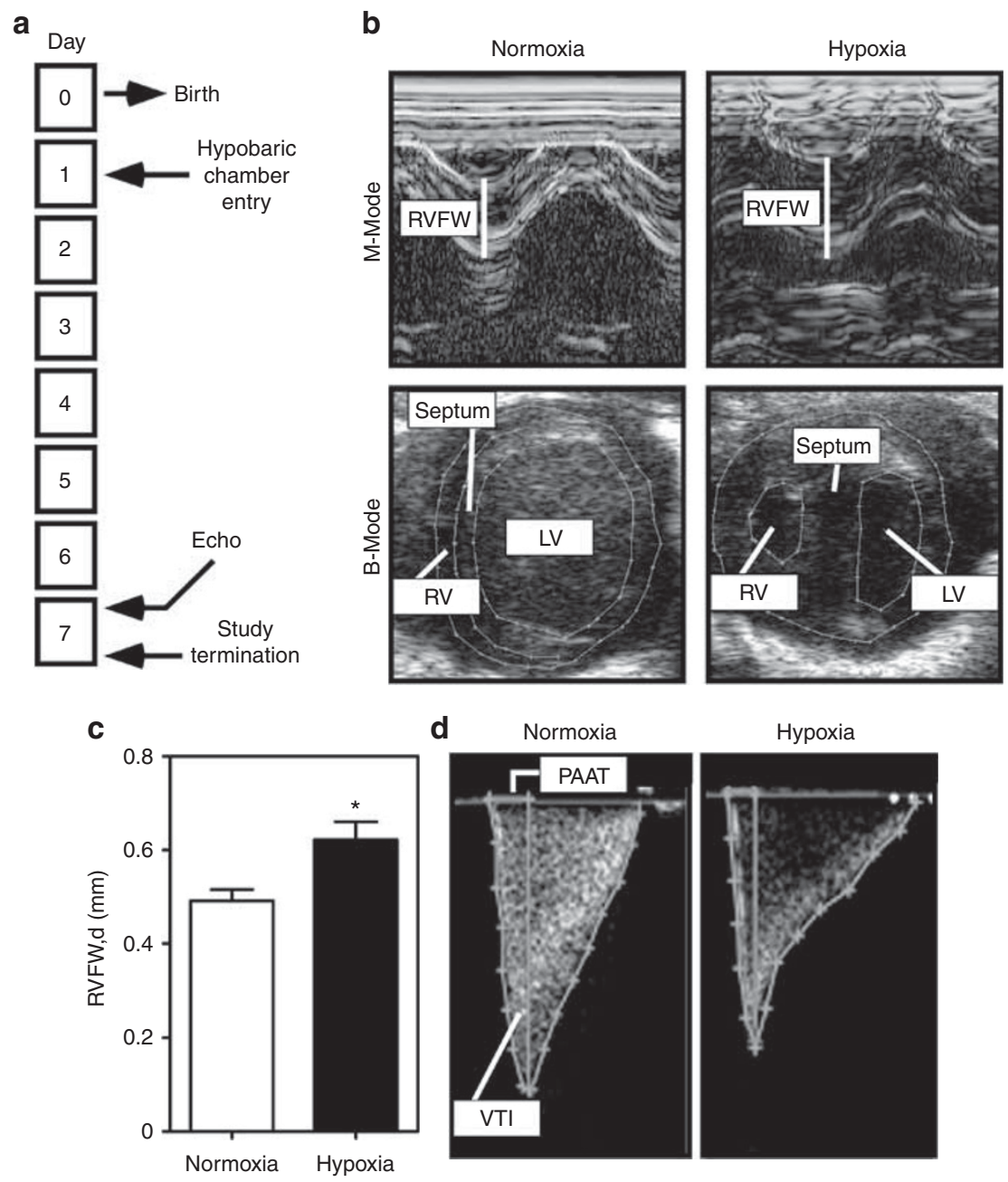

d
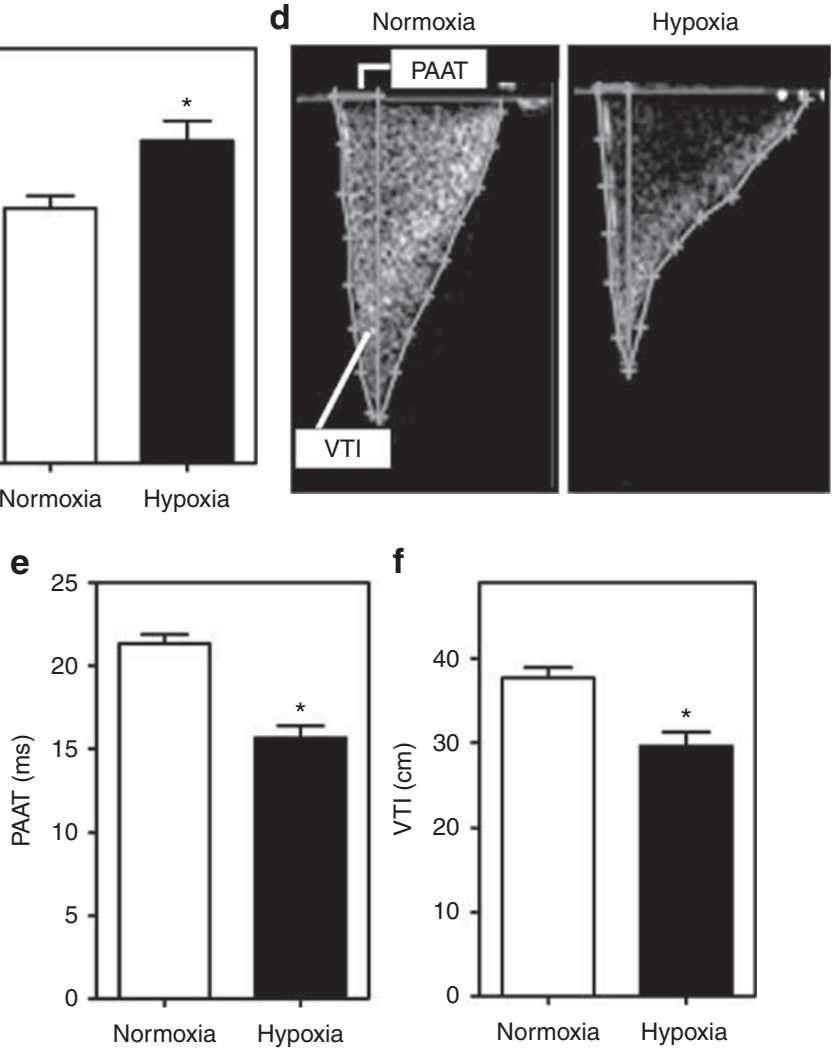

Figure 3. Echocardiographic analyses of right ventricular hypertrophy and pulmonary hypertension in hypoxic neonatal rats. (a) Study design. (b) Representative M-mode and B-mode images of hearts from neonatal rats exposed to hypobaric hypoxia or normoxic conditions for 7 days. (c) RVfree wall thickness, diastole: quantitative assessment of RV-free wall (RVFW) thickness demonstrates increased thickness in the hypoxic rats; ${ }^{*} P<0.05$ vs. controls. (d) Representative Doppler images of pulmonary blood flow in normoxic and hypoxic neonatal rats. (e) Pulmonary artery acceleration time (PAAT) is significantly decreased in the hypoxic neonatal rats compared with control. (f) Pulmonary valve velocity-time integral (VTI) is also significantly decreased in hypoxic neonatal rats compared with control. Results are displayed as the mean with $\mathrm{SE}$. $N=11$ per condition; $P<0.05$ vs. controls. RV, right ventricle.

rats. Significant increases in class I HDAC and class IIb HDAC catalytic activity were observed in hypertrophic RVs, which was consistent with pediatric SVs. However, unlike pediatric SVs, there was no difference in class IIa HDAC catalytic activity in hypoxic RVs (Figure 5a). Increases in class I and class IIb HDAC activity correlated with the abundance of HDAC1, HDAC2, HDAC3, and HDAC6 in RVs of hypoxic neonatal rats (Figure $5 b$ ). 
a

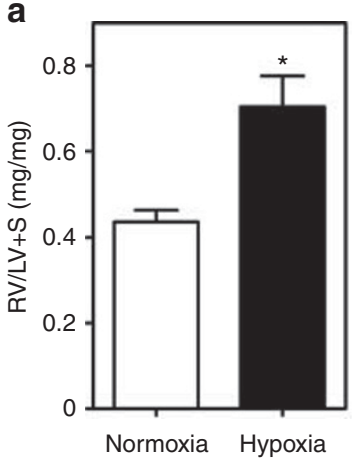

b

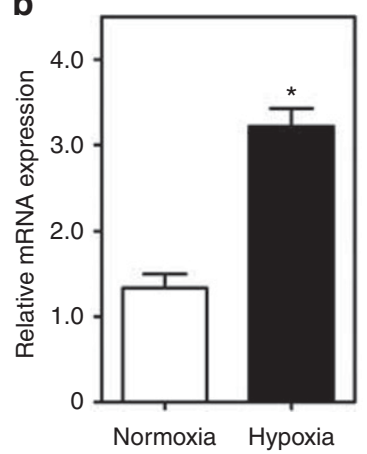

C

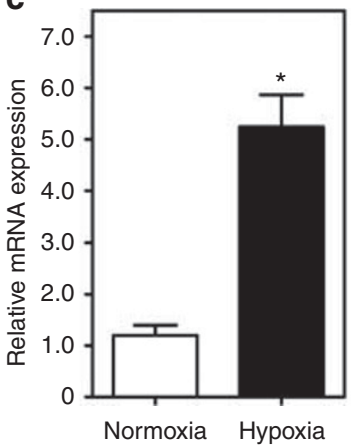

d

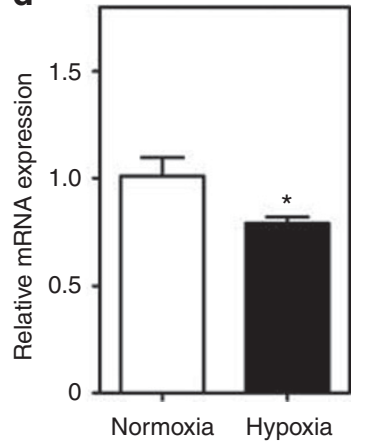

Figure 4. Pathological RV remodeling in neonatal rats exposed to hypoxia. (a) RV hypertrophy was assessed by comparing weights of RV vs. LV +septum (S). The RV/LV+septum ratio was higher in the hypoxic rats. (b) ANP, (c) B-type natriuretic peptide (BNP), and (d) sarcoplasmic reticulum calcium ATPase 2a (Serca2a) mRNA expression were determined by quantitative PCR. ANP and BNP expression was increased, whereas Serca2a expression was decreased in the hypoxic animals. Results are presented as the mean with SE. $N=3$ (normoxia), $N=6$ (hypoxia); ${ }^{*} P<0.05$ vs. control. ANP, atrial natriuretic peptide; LV, left ventricle; RV, right ventricle; SV, single ventricle.

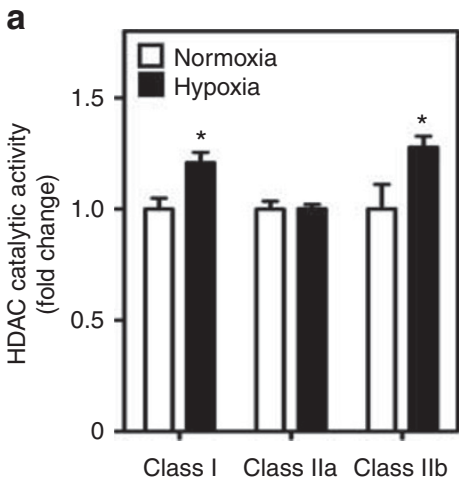

\section{b}

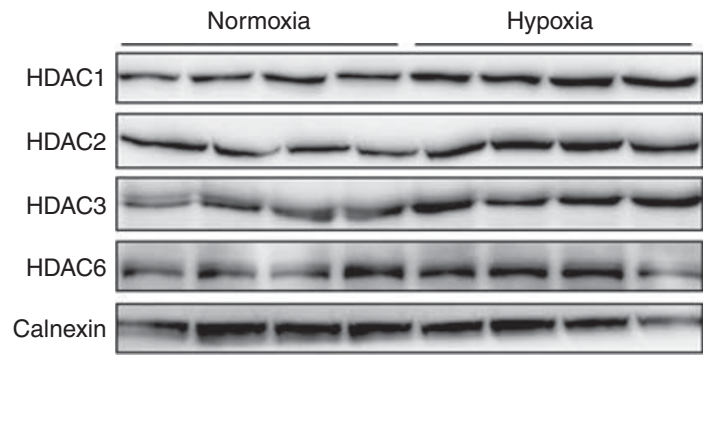

Figure 5. Activation of HDACs during pathological RV remodeling in neonatal rats. (a) HDAC catalytic activity was quantified in RV homogenates from neonatal rats exposed to hypobaric hypoxia $(N=6)$ or normoxic $(N=3)$ conditions for 7 days. Class I and Ilb activity was increased in the RV of the hypoxic rats. Results are displayed as the mean with $\mathrm{SE}$; ${ }^{*} P<0.05$ vs. normoxic controls. (b) Immunoblot analysis was performed with the same rat RV homogenates used for the HDAC catalytic activity assays employing antibodies specific for class I HDACs (HDAC1-3) and class IIb HDAC6. HDAC 1, 2, 3, and 6 protein expression was increased in the RV of the hypoxic rats. HDAC, histone deacetylase; RV, right ventricle.

\section{DISCUSSION}

Pharmacotherapy to prevent or treat myocardial failure in patients with SV physiology does not exist, underscoring the need for research to define the molecular mechanisms of this complex congenital heart lesion (4). Treatment of adult HF has markedly advanced over the past several decades; yet many of the drugs used to treat adult HF have not been proven to be beneficial in treating pediatric HF (21). Part of the challenge lies in the fact that pediatric HF is a much more heterogeneous disease than HF in adults, with a low incidence of ischemic disease. Pediatric HF ranges from idiopathic dilated cardiomyopathy, which is the leading indication for heart transplant in children, to SV heart disease, the leading indication for transplant in infants (22). There are inherent differences in myocardial adaptation in pediatric vs. adult HF, including altered gene, protein, adrenergic receptor, and microRNA expression, as well as protein phosphorylation and enzyme activity $(18,23-25)$. In addition, children are a vulnerable population, limiting the ability to perform the invasive studies that have been so useful in advancing the care of adults with HF. This study was designed to characterize the adaptations occurring in myocardial HDACs in the pediatric population with a SV, and to develop and perform initial characterization of an animal model that could serve to facilitate future mechanistic studies and drug development relevant to RV remodeling in the young.

We found that the catalytic activity of class I, IIa, and IIb HDACs is elevated in RVs of children with SV disease compared with NF controls (Figure 1b). For class I and IIa HDACs, enhanced catalytic activity correlated with increased levels of HDAC isoform protein expression (class I HDACs $-1,-2$, and -3 ; class IIa HDAC5; Figure 2). In contrast, activation of class IIb HDAC activity occurred independently of altered expression of the predominant isoform within this class, HDAC6. In this regard, multiple post-translational 


\section{Articles | Blakeslee et al.}

mechanisms for regulation of HDAC6 activity have been described in non-cardiac cells. For example, phosphorylation of HDAC6 by glycogen synthase kinase-3 $\beta$ was shown to activate HDAC6 catalytic activity in neurons (26). Elucidation of the mechanisms controlling HDAC catalytic activity in SV disease awaits further investigation.

The molecular mechanisms by which HDACs contribute to adult pressure overload-induced LV hypertrophy, myocardial interstitial fibrosis, cardiac inflammation, $\mathrm{PH}$, and $\mathrm{RV}$ remodeling are only beginning to be understood (27-29). HDAC inhibition is effective in improving HF symptoms in animal models of spontaneously hypertensive rats (30), mouse models of myocardial infarction/ischemia-reperfusion (31), and an angiotensin II-infusion model of cardiac fibrosis (12). Consistent with our hypothesis, the class I HDAC inhibitor apicidin attenuated hypoxia-induced $\mathrm{RVH}$ and pulmonary vascular remodeling in neonatal mice (32). A recent proteomic study using 19 different HDAC inhibitors in three different human cell lines demonstrated the complexity of individual small-molecule effects on the acetylome, with differential effects on the magnitude of acetylation signal and specific acetylation targets between compounds (33). Because of these findings, selection of the class of HDAC inhibitor and specific small molecule for therapeutic development requires careful consideration.

In combination with our previously described abnormalities in the beta-AR system (18), elevated HDAC catalytic activity and expression in the RVs of children with a SV suggest that SV hearts are undergoing molecular remodeling. Consistent with these findings, clinically the single RV does not tolerate chronic pressure overload indefinitely, and patients remain at risk for the development of ventricular failure.

Although prenatal models of SV exist (34-36), a postnatal animal model is not feasible because of complexities of maintaining the SV circulation after birth. Therefore, we developed a hypoxic neonatal rat model that reasonably recapitulates some of the echocardiographic and molecular signatures of pediatric SV. Children born with a SV are hypoxic until the third stage of surgical palliation (Fontan) is completed at 2-5 years of age and systemic afterload on the $\mathrm{RV}$ is present throughout their lifespan, with hypertrophy essentially a universal finding. Hypoxia in neonatal rats results in $\mathrm{PH}$ and $\mathrm{RVH}$ by echocardiographic and morphometric assessment, altered myocardial gene expression, with partial recapitulation of the fetal gene program, elevated HDAC class I and IIb activity, and elevated HDAC protein expression. From both a physiologic and molecular perspective, the hypoxic neonatal rat model has similarities to the human SV condition that support its use for future mechanistic studies of RV remodeling, as well as for therapeutic development. Although HDAC IIa activity was not increased in the rat RV as it was in the pediatric SV, whether this difference is clinically significant is unknown. Interestingly, HDAC 4 is associated with the cardiac sarcomere and may have a role in regulating cardiac contractility (37), warranting future study. The use of echocardiography in this model not only provides translational utility but also allows for longitudinal investigation. $\mathrm{RVH}$ and failure are not uncommon in the pediatric cardiovascular disease population, and this model has potential to affect a myriad of populations beyond SV. PH, intrinsic lung disease, and various forms of biventricular congenital heart disease (e.g., tetralogy of Fallot, Ebstein's anomaly) also result in pediatric RV remodeling and failure.

There are important limitations of this study. The tissue bank-based aspect of this study is inherently cross-sectional, and proof of mechanistic associations based on our results is not possible. Because of the rarity of this form of congenital heart disease, and thus the limited tissue available for study, it is not possible for us to determine the influence of age, prior surgical procedures, medications, clinical status of the patient, or the temporal relationship of gene expression changes on our findings. We recognize that we have not developed a rodent model of SV. However, many of the findings in this study show consistent changes in phenotype and molecular signatures of hypoxic neonatal rats and patients born with a SV vs. their respective controls. This model could be used as a platform for the study of mechanisms of pressure-overloaded $\mathrm{RV}$ failure in the young and determination of the influence of aging and development vs. disease on myocardial adaptations.

In conclusion, although HDACs are ubiquitously expressed and are essential enzymes, HDAC inhibitors are surprisingly well tolerated (38-40). There is an evolving body of literature, suggesting that HDAC inhibition has value for the treatment of adult cardiovascular diseases (14). The results of this study demonstrate that HDACs are altered in the ventricle of young children with SV heart disease. Given the challenges of studying a rare disease in a vulnerable population, the hypoxic neonatal rat represents a reasonable platform for extending preclinical studies to explore mechanisms of disease and investigate novel therapeutics, such as HDAC inhibitors, in preventing or limiting pathologic RV remodeling.

\section{STATEMENT OF FINANCIAL SUPPORT}

This work was supported by NIH R21 HL113846 to S.D.M., T.A.M and C.S.L. S.D.M. was also supported by NIH (R01 HL126928), the Addison Scott Memorial Fund, the Boedecker Foundation, the Nair Family, and the Millisor Chair in Pediatric Heart Disease. T.A.M. was also supported by NIH (HL116848, HL127240 and AG043822) and the American Heart Association (GIA14510001 and 16SFRN31400013). W.W.B was supported by the University of Colorado-Denver Pharmacology Program NIH T32 Training Grant (GM007635).

Disclosure: The authors declare no conflict of interest.

\section{REFERENCES}

1. Correa-Villasenor A, Ferencz C, Loffredo C, Magee C. Paternal exposures and cardiovascular malformations. The Baltimore-Washington Infant Study Group. J Expo Anal Environ Epidemiol 1993;3 (Suppl 1): 173-85.

2. Ohye RG, Sleeper LA, Mahony L, et al. Comparison of shunt types in the Norwood procedure for single-ventricle lesions. N Engl J Med 2010;362: 1980-92.

3. Kulkarni A, Neugebauer R, Lo Y, et al. Outcomes and risk factors for listing for heart transplantation after the Norwood procedure: an analysis 


\section{HDAC adaptation in the right ventricle Articles}

of the single ventricle reconstruction trial. J Heart Lung Transplant 2016;35:306-11.

4. Kirk R, Dipchand AI, Rosenthal DN, et al. The International Society for Heart and Lung Transplantation Guidelines for the management of pediatric heart failure: Executive summary. [Corrected]. J Heart Lung Transplant 2014;33:888-909.

5. Frommelt PC, Gerstenberger E, Cnota JF, et al. Impact of initial shunt type on cardiac size and function in children with single right ventricle anomalies before the Fontan procedure: the single ventricle reconstruction extension trial. J Am Coll Cardiol 2014;64:2026-35.

6. Newburger JW, Sleeper LA, Frommelt PC, et al. Transplantation-free survival and interventions at 3 years in the single ventricle reconstruction trial. Circulation 2014;129:2013-20.

7. Lu J, McKinsey TA, Nicol RL, Olson EN. Signal-dependent activation of the MEF2 transcription factor by dissociation from histone deacetylases. Proc Natl Acad Sci USA 2000;97:4070-5.

8. Tanno M, Kuno A, Horio Y, Miura T. Emerging beneficial roles of sirtuins in heart failure. Basic Res Cardiol 2012;107:273.

9. Demos-Davies KM, Ferguson BS, Cavasin MA, et al. HDAC6 contributes to pathological responses of heart and skeletal muscle to chronic angiotensin-II signaling. Am J Physiol Heart Circ Physiol 2014;307: H252-8.

10. Mani SK, Kern CB, Kimbrough D, et al. Inhibition of class I histone deacetylase activity represses matrix metalloproteinase-2 and -9 expression and preserves LV function postmyocardial infarction. Am J Physiol Heart Circ Physiol 2015;308:H1391-401.

11. Cao DJ, Wang ZV, Battiprolu PK, et al. Histone deacetylase (HDAC) inhibitors attenuate cardiac hypertrophy by suppressing autophagy. Proc Natl Acad Sci USA 2011;108:4123-8.

12. Williams SM, Golden-Mason L, Ferguson BS, et al. Class I HDACs regulate angiotensin II-dependent cardiac fibrosis via fibroblasts and circulating fibrocytes. J Mol Cell Cardiol 2014;67:112-25.

13. Antos CL, McKinsey TA, Dreitz M, et al. Dose-dependent blockade to cardiomyocyte hypertrophy by histone deacetylase inhibitors. J Biol Chem 2003;278:28930-7.

14. McKinsey TA. Therapeutic potential for HDAC inhibitors in the heart. Annu Rev Pharmacol Toxicol 2012;52:303-19.

15. Lemon DD, Horn TR, Cavasin MA, et al. Cardiac HDAC6 catalytic activity is induced in response to chronic hypertension. J Mol Cell Cardiol 2011;51:41-50.

16. Blakeslee WW, Wysoczynski CL, Fritz KS, Nyborg JK, Churchill ME, McKinsey TA. Class I HDAC inhibition stimulates cardiac protein SUMOylation through a post-translational mechanism. Cell Signal 2014;26:2912-0.

17. Cavasin MA, Demos-Davies K, Horn TR, et al. Selective class I histone deacetylase inhibition suppresses hypoxia-induced cardiopulmonary remodeling through an antiproliferative mechanism. Circ Res 2012;110: $739-48$.

18. Miyamoto SD, Stauffer BL, Polk J, et al. Gene expression and beta-adrenergic signaling are altered in hypoplastic left heart syndrome. J Heart Lung Transplant 2014;33:785-93.

19. Cevik A, Kula S, Olgunturk R, et al. Assessment of pulmonary arterial hypertension and vascular resistance by measurements of the pulmonary arterial flow velocity curve in the absence of a measurable tricuspid regurgitant velocity in childhood congenital heart disease. Pediatr Cardiol 2013;34:646-55.

20. Vlahos AP, Feinstein JA, Schiller NB, Silverman NH. Extension of Doppler-derived echocardiographic measures of pulmonary vascular resistance to patients with moderate or severe pulmonary vascular disease. J Am Soc Echocardiogr 2008;21:711-4.
21. Rossano JW, Shaddy RE. Update on pharmacological heart failure therapies in children: do adult medications work in children and if not, why not? Circulation 2014;129:607-12.

22. Rossano JW, Dipchand AI, Edwards LB, et al. The Registry of the International Society for Heart and Lung Transplantation: Nineteenth Pediatric Heart Transplantation Report-2016; Focus Theme: primary diagnostic indications for transplant. J Heart Lung Transplant 2016;35:1185-95.

23. Miyamoto SD, Karimpour-Fard A, Peterson V, et al. Circulating microRNA as a biomarker for recovery in pediatric dilated cardiomyopathy. J Heart Lung Transplant 2015;34:724-33.

24. Miyamoto SD, Stauffer BL, Nakano S, et al. Beta-adrenergic adaptation in paediatric idiopathic dilated cardiomyopathy. Eur Heart J 2014;35:33-41.

25. Nakano SJ, Miyamoto SD, Movsesian M, Nelson P, Stauffer BL, Sucharov CC. Age-related differences in phosphodiesterase activity and effects of chronic phosphodiesterase inhibition in idiopathic dilated cardiomyopathy. Circ Heart Fail 2015;8:57-63.

26. Chen S, Owens GC, Makarenkova H, Edelman DB. HDAC6 regulates mitochondrial transport in hippocampal neurons. PLoS ONE 2010;5: e10848.

27. Cavasin MA, Stenmark KR, McKinsey TA. Emerging roles for histone deacetylases in pulmonary hypertension and right ventricular remodeling (2013 Grover Conference series). Pulm Circ 2015;5:63-72.

28. Gillette TG, Hill JA. Readers, writers, and erasers: chromatin as the whiteboard of heart disease. Circ Res 2015;116:1245-53.

29. Schuetze KB, McKinsey TA, Long CS. Targeting cardiac fibroblasts to treat fibrosis of the heart: focus on HDACs. J Mol Cell Cardiol 2014;70:100-7.

30. Cardinale JP, Sriramula S, Pariaut R, et al. HDAC inhibition attenuates inflammatory, hypertrophic, and hypertensive responses in spontaneously hypertensive rats. Hypertension 2010;56:437-4.

31. Granger A, Abdullah I, Huebner F, et al. Histone deacetylase inhibition reduces myocardial ischemia-reperfusion injury in mice. FASEB J 2008;22:3549-60.

32. Yang Q, Sun M, Ramchandran R, Raj JU. IGF-1 signaling in neonatal hypoxia-induced pulmonary hypertension: role of epigenetic regulation. Vascul Pharmacol 2015;73:20-31.

33. Scholz C, Weinert BT, Wagner SA, et al. Acetylation site specificities of lysine deacetylase inhibitors in human cells. Nat Biotechnol 2015;33: 415-23.

34. deAlmeida A, Sedmera D. Fibroblast growth factor-2 regulates proliferation of cardiac myocytes in normal and hypoplastic left ventricles in the developing chick. Cardiol Young 2009;19:159-69.

35. Kowalski WJ, Teslovich NC, Menon PG, Tinney JP, Keller BB, Pekkan K. Left atrial ligation alters intracardiac flow patterns and the biomechanical landscape in the chick embryo. Dev Dyn 2014;243:652-2.

36. Sedmera D, Hu N, Weiss KM, Keller BB, Denslow S, Thompson RP. Cellular changes in experimental left heart hypoplasia. Anat Rec 2002;267:137-45.

37. Gupta MP, Samant SA, Smith SH, Shroff SG. HDAC4 and PCAF bind to cardiac sarcomeres and play a role in regulating myofilament contractile activity. J Biol Chem 2008;283:10135-46.

38. Klimek VM, Fircanis S, Maslak P, et al. Tolerability, pharmacodynamics, and pharmacokinetics studies of depsipeptide (romidepsin) in patients with acute myelogenous leukemia or advanced myelodysplastic syndromes. Clin Cancer Res 2008;14:826-32.

39. Marks PA. The clinical development of histone deacetylase inhibitors as targeted anticancer drugs. Expert Opin Investig Drugs 2010;19:1049-66.

40. O'Connor OA, Heaney ML, Schwartz L, et al. Clinical experience with intravenous and oral formulations of the novel histone deacetylase inhibitor suberoylanilide hydroxamic acid in patients with advanced hematologic malignancies. J Clin Oncol 2006;24:166-73. 